\title{
ACCRETION FLOWS IN HIGH-MASS STAR FORMATION
}

\author{
Eric Keto \\ Lawrence Livermore National Laboratory \\ Institute of Geophysics and Planetary Physics
}

\begin{abstract}
We compare observed and simulated images of the accretion flows associated with high mass star formation in the regions G10.6-0.4 and DR21. We describe, as a result of the comparison, the temperature, density, and velocity fields. Our results indicate that the G10.6-0.4 cloud core is strongly condensed and has approximately equal velocities in rotation and infall at its current evolutionary state. The rapid collapse and lack of rotational support suggests that significant angular momentum transfer is occuring over scales at least as large as those observed $(0.5 \mathrm{pc})$. A milligauss magnetic field would have sufficient energy to supply the required braking torque of $10^{47}$ ergs. The DR21 core shows approximately spherically symmetric radial accretion with no detectable rotation. Unlike the G10.6-0.4 core, the DR21 core does not contain an embedded HII region. Thus this core may represent a molecular cloud condensation undergoing gravitational collapse and accretion just prior to the formation of massive stars.
\end{abstract}

\section{RADIATIVE TRANSFER}

For comparison of any model with observations we need to compute a three dimensional image of the line brightness where two of the three dimensions are the $x$ and $y$ map positions, and the third is the line-of-sight velocity. We adopt the following simple scheme. From the model $\mathrm{H}_{2}$ density, temperature, and fractional abundance of our molecular tracer we compute the source function and opacity assuming LTE. The local line profile is specified by the thermal and turbulent broadening and the model line-of-sight velocity. The line brightness at a particular frequency is determined by integrating the radiative transfer equation along the line of sight. The resulting image is then convolved with the beam pattern and autocorrelator response appropriate to the observing apparatus.

\section{G10.6-0.4}

Spectral line observations of the dense gas immediately around the G10.6-0.4 HII region suggest infall and rotation consistent with gravitational collapse in the potential 
well of the gas and stars. The flow represents a continuation beyond the onset of nuclear burning of the original accretion responsible for star-formation. The rotational and infall velocities increase with decreasing radius suggesting spin-up and accelerating infall. Comparison of the $\mathrm{NH}_{3}(1,1)$ to $\mathrm{NH}_{3}(3,3)$ line ratios indicates that the molecular cloud core is heated by dust reradiation of the UV continuum from the newly formed stars and that the density is centrally condensed (Ho and Haschick 1986, Keto et al. 1987, 1988).

Our observations of G10.6-0.4 include four data sets. We observed the $\mathrm{NH}_{3}(1,1)$ and $(3,3)$ rotational states using a compact configuration (D) of the VLA to achieve $10^{\prime \prime}$ resolution, and an extended configuration (B) for $0.3^{\prime \prime}$ resolution. In figures $1 \mathrm{a}$ and $2 \mathrm{a}$, we plot the line brightness as a function of velocity and position in the equatorial plane of the rotating gas from two of these data sets. In figures $1 \mathrm{~b}$ and $2 \mathrm{~b}$, we show plots generated from our model. Table 1 lists the equations and parameters which constitute the model. The model has been arbitrarily chosen on the basis of mathematical simplicity guided by the analysis of the observations. The model is purely diagnostic in the sense that it is designed to match the observations rather than describe the physics of the accretion flow.

Table 1. Model Parameters for G10.6-0.4

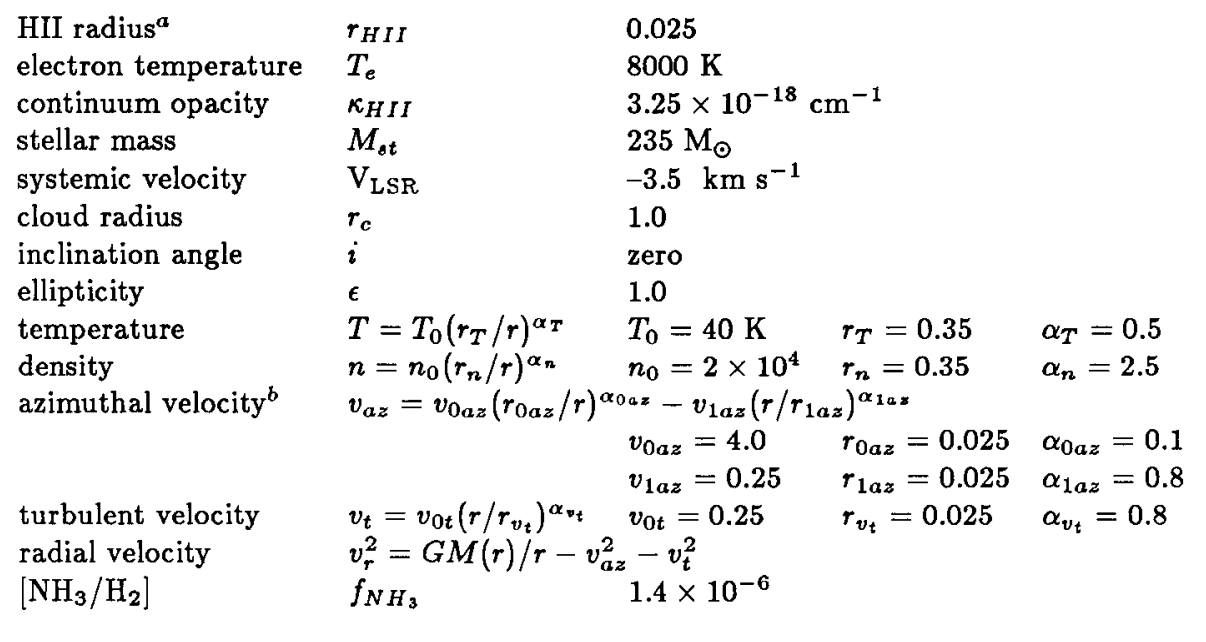

${ }^{a}$ All radii are in pc.

${ }^{b}$ All velocities are in $\mathrm{km} \mathrm{s}^{-1}$.

Figure 3a shows a vector plot of the modeled velocities in the equatorial plane and Fig. $3 b$, the three velocity components with radius. The gas spirals into the center quite rapidly, completing less than one orbit over the two decades in radial distances accessible by the observations. The failure to achieve rotational support 

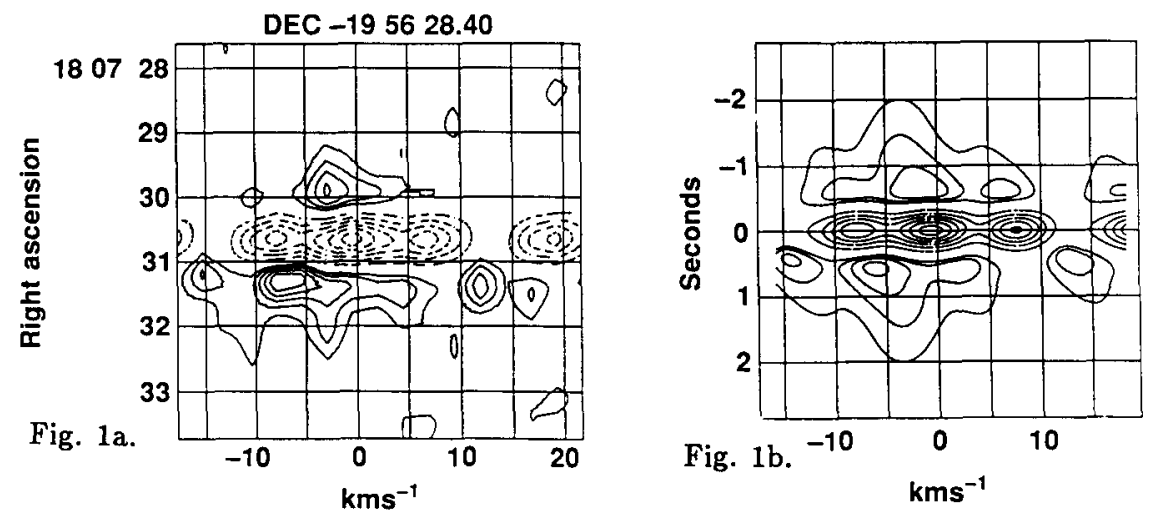

Fig. 1a. Position-velocity plot of the $\mathrm{NH}_{3}(1,1)$ line brightness in the equatorial plane of G10.6-0.4. The resolution is $10^{\prime \prime}$ and $2.4 \mathrm{~km} \mathrm{~s}^{-1}$. The contour interval is 50 $\mathrm{mJy} /$ beam in emission and $200 \mathrm{mJy} /$ beam in absorption (dashed contours). The multiple components are due to the hyperfine structure of $\mathrm{NH}_{3}$. Fig. 1b. Model position-velocity plot. The contour interval is 8.5 in degrees $\mathrm{K}$ in emission and 21 $\mathrm{K}$ in absorption.
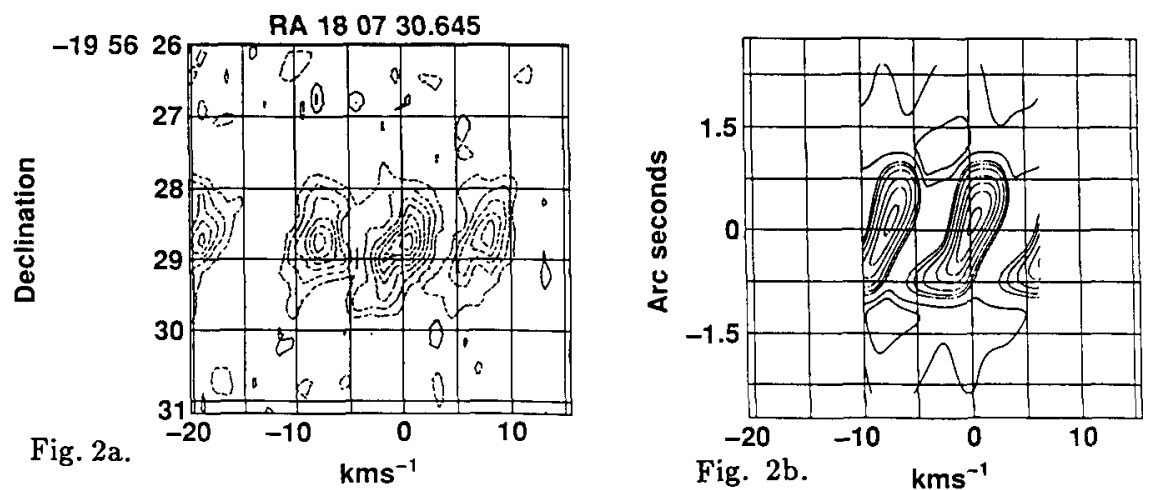

Fig. 2a. Position-velocity diagram of the $\mathrm{NH}_{3}(1,1)$ line at $0.4^{\prime \prime}$ and $1.2 \mathrm{~km} \mathrm{~s}^{-1}$ resolution. The contour interval is $-30 \mathrm{mJy} /$ beam. Fig. $2 \mathrm{~b}$. Model position-velocity diagram. The contours are $-625,-500,-375,-250,-125,-62,-31,12.5,25 \mathrm{~K}$. Only a portion of Fig. $2 \mathrm{a}$ has been modeled.

suggests either collapse from a state of negligibly low angular momentum, or more likely, strong angular momentum braking. The time rate of change of the angular momentum may be estimated as $d L / d t=m v_{r}\left(v_{a z 1} r_{1}-v_{a z 2} r_{2}\right) /\left(r_{1}-r_{2}\right)$. This estimate strictly applies only assuming a steady state velocity field which we know cannot be correct. Nevertheless, the required torque is about $5 \times 10^{46}$ ergs from 0.35 to $0.1 \mathrm{pc}$ and $10^{47}$ ergs from 0.1 to $0.025 \mathrm{pc}$. The average field strength, $\left\langle B^{2} / 8 \pi\right\rangle$, required to equal this torque is $0.5 \mathrm{mG}$ within $0.35 \mathrm{pc}$ and a factor of ten higher within $0.1 \mathrm{pc}$. The required field strengths are within the range measured by Zeeman splitting of $\mathrm{OH}$ masers around similar HII regions and are also consistent with the increase expected from flux freezing in the condensed core. 


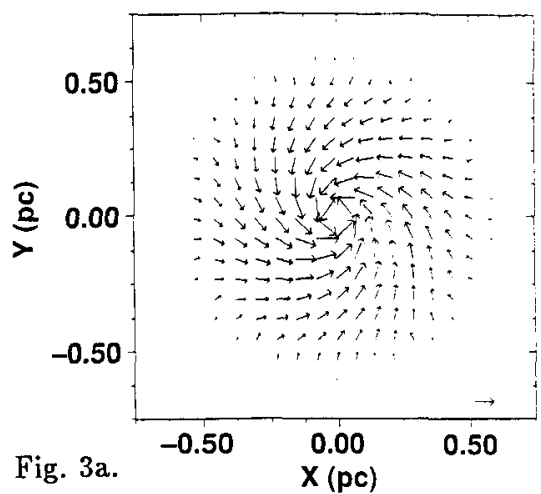

Fig. 3a. The velocity directions and magnitudes in the equatorial plane of the G10.6-0.4.

The arrow lower right is $1.3 \mathrm{~km} \mathrm{~s}^{-1}$. $3 b$. The three velocity components used in the model and the gravitational energy in $\mathrm{km} \mathrm{s}^{-1}$.

DR21

Continuum observations show that massive star forming regions typically contain clusters of compact and ultracompact HII regions of a range of sizes and ages. Thus there should be dense cores in the molecular cloud surrounding these newly formed HII regions which are at an earlier evolutionary stage. This stage would be characterized by an accretion flow similar to that observed around G10.6-0.4 but existing prior to the formation of ZAMS stars and the ionization of the immediate molecular gas.

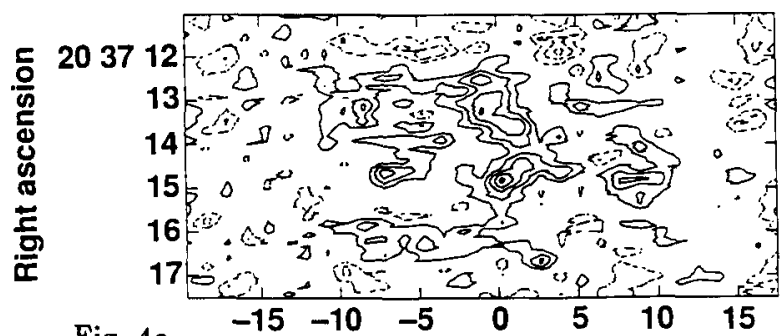

Fig. $4 a$.

$$
\mathrm{Kms}^{-1}
$$

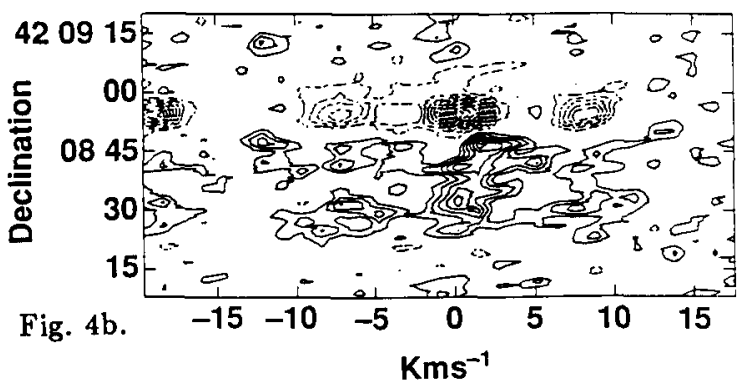

Fig. 4a. Position-velocity along an east-west line at $\operatorname{Dec}(1950)=42^{\circ} 08^{\prime} 37^{\prime \prime}$ near DR21. The contour interval is $10 \mathrm{mJy} /$ beam. $4 b$. Position-velocity along a north-south line at $\mathrm{RA}(1950)=20^{\mathrm{h}} 37^{\mathrm{m}} 13 .{ }^{\prime \prime} 7$ The contour level in absorption is $20 \mathrm{mJy} /$ beam. 
Observations in the $(1,1)$ and $(3,3)$ rotational lines of $\mathrm{NH}_{3}$ suggest that a dense concentration of gas to the south of the DR21 cluster of HII regions is such an isolated molecular cloud core undergoing gravitational collapse in the earliest stages of star formation.

In Figures $4 \mathrm{a}$ and $4 \mathrm{~b}$ we show velocity-position diagrams made along east-west and north-south lines across the molecular core. The prominent curved structures seen in Fig. $4 \mathrm{a}$ in emission and in $4 \mathrm{~b}$, if the northern portion of the core seen in absorption is taken into account, are indicative of projection effects associated with radial flow. The fact that the projection effects are seen in perpendicular orientations implies that the radial flow is approximately spherically symmetric. The position of the most redshifted velocity defines the collapse center, $\operatorname{RA}(1950)=20^{\mathrm{h}} 37^{\mathrm{m}} 13 . " 7, \operatorname{Dec}(1950)=42^{\circ} 08^{\prime} 37^{\prime \prime}$.

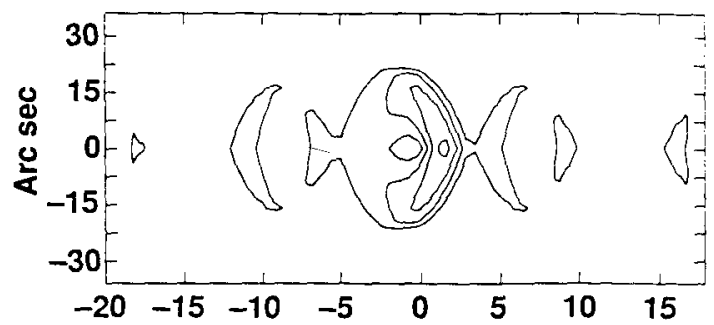

Fig. 5.

$\mathrm{Kms}^{-1}$

Fig. 5. Model velocity-position diagram to be compared with Figures $4 \mathrm{a}$ and $4 \mathrm{~b}$.

Figure 5 shows a model velocity-position plot generated assuming LTE radiative transfer and a spherical core with constant radial velocity of $2.5 \mathrm{~km} \mathrm{~s}^{-1}, \mathrm{n}\left(\mathrm{H}_{2}\right)$ density of $10^{5} \mathrm{~cm}^{-3}$, kinetic temperature of $40 \mathrm{~K}$, and linewidth of $1.0 \mathrm{~km} \mathrm{~s}^{-1}$. The model includes a foreground cloud with constant line of sight velocity of $2.5 \mathrm{~km} \mathrm{~s}^{-1}, \mathrm{n}\left(\mathrm{H}_{2}\right)$ density of $10^{4} \mathrm{~cm}^{-3}$, temperature of $10 \mathrm{~K}$, and linewidth of $2.0 \mathrm{~km} \mathrm{~s}^{-1}$. In the absence of this cold foreground cloud, one should expect to see both the front and back hemispheres of the spherically symmetric radial flow. This would manifest itself on the velocity-position plots as a mirror image of the curved structure around $\mathrm{V}_{\mathrm{LSR}}=+2.5$ $\mathrm{km} \mathrm{s}^{-1}$, but at a $V_{L S R}$ of about $-2.5 \mathrm{~km} \mathrm{~s}^{-1}$. We suggest that the missing emission at $-2.5 \mathrm{~km} \mathrm{~s}^{-1}$ is absorbed by the large scale, cold, foreground cloud. This cloud is not apparent in our data in emission because structure larger than $75^{\prime \prime}$, or twice the southern core diameter, will be resolved out. However, we do detect a component at this $V_{L S R}$ in absorption. In addition, there is considerable evidence from single dish and lower angular resolution observations for a large scale molecular cloud detected in $\mathrm{NH}_{3}(1,1)$ and $(2,2), \mathrm{OH}, \mathrm{HCN}$, and $\mathrm{H}_{2} \mathrm{CO}$ at -2 to $-3 \mathrm{~km} \mathrm{~s}^{-1}$ (Dickel et al. 1978; Matsakis et al. 1981; Guilloteau et al. 1983; Johnston et al. 1984; Dickel, Ho, and Wright 1985). Since the modeled flow is spherically symmetric, Fig. 5 should apply equally well to both orientations in Fig. 4 provided that the fact that the northern portion of the core is seen in absorption in Fig. $4 \mathrm{~b}$ is taken into account. 
From these observations it is not possible to determine whether we are observing the redshifted front or back hemisphere of the radial flow. However, the observed radial velocity of $2.5 \mathrm{~km} \mathrm{~s}^{-1}$ at $0.2 \mathrm{pc}$ is consistent with infall in the self-gravity of a $290 \mathrm{M}_{\odot}$ core. The mass of the core estimated independently from the column density of $\mathrm{NH}_{3}$ is $270 \mathrm{M}_{\odot}$ to an accuracy of a factor of two.

Work performed under the auspices of U.S. Department of Energy at Lawrence Livermore National Laboratory under contract number W-7405-ENG-48.

\section{REFERENCES}

Dickel, H. R., Ho, P. T. P., and Wright, M. C. H., 1985, Ap. J., 290, 256.

Guilloteau, S., Wilson, T. L., Martin, R. N., Batrla', W., and Pauls, T. A., 1983 Astr. Ap., 124, 322.

Ho, P. T. P., and Haschick, A. D. 1986, Ap. J., 304, 501.

Johnston, K.J., Henkel, C., and Wilson, T.L., 1984 Ap. J. (Letters), 285 , L85.

Keto, E. R., Ho, P. T. P., and Haschick, A. D., 1987a, Ap. J., 318, 712.

Keto, E. R., Ho, P. T. P., and Haschick, A. D., 1988, Ap. J., 324, 920.

Matsakis, D. N., Hjalmarson, A., Palmer, P., Cheung, A. C., and Townes, C. H., 1981, Ap. J. (Letters), 250, L89. 